\title{
A NOTE ON $N_{K}$ CONFIGURATIONS AND THEOREMS IN PROJECTIVE SPACE
}

\author{
David G. GLYNN
}

\begin{abstract}
A method of embedding $n_{k}$ configurations into projective space of $k-1$ dimensions is given. It breaks into the easy problem of finding a rooted spanning tree of the associated Levi graph. Also it is shown how to obtain a "complementary" $n_{n-k}$ "theorem" about projective space (over a field or skew-field $F$ ) from any $n_{k}$ theorem over $F$. Some elementary matroid theory is used, but with an explanation suitable for most people. Various examples are mentioned, including the planar configurations: Fano $7_{3}$, Pappus 93 , Desargues $10_{3}$ (also in 3d-space), Möbius 84 (in $3 \mathrm{~d}$-space), and the resulting 74 in $3 \mathrm{~d}$-space, $9_{6}$ in $5 \mathrm{~d}$-space, and $10_{7}$ in $6 \mathrm{~d}$-space. (The Möbius configuration is self-complementary.) There are some $n_{k}$ configurations that are not embeddable in certain projective spaces, and these will be taken to similarly not embeddable configurations by complementation. Finally, there is a list of open questions.
\end{abstract}

\section{INTRODUCTION}

DEFINITION 1: A combinatorial $n_{k}$ configuration is an incidence structure $(P, B, I)$ with a set $P$ of $n$ points and a set $B$ of $n$ blocks, (which may be considered to be subsets of $P$ ), such that each block contains $k$ of the points, and each point is on $k$ of the blocks. A point $p \in P$ is on a block $b \in B$ if and only if $p I b$, where $I$ is the "incidence relation". N.B. We usually omit the term "combinatorial".

For us the problem of embedding these $n_{k}$ configurations is to find $n$ points and $n$ hyperplanes in $k-1$ dimensional projective space (coordinatised by a field or skewfield) such that incidence in the configuration is preserved. (A skew-field satisfies the same axioms as a field but with a multiplication that need not be commutative.) Some extra incidences may appear but usually these are ignored. Note that, to the contrary, some authors appear to require that an embedding, or realisaton, may not have any extra incidences at all. See for example the survey by Gropp [7], and the $16_{3}$ of Figure 8 there. Further, a "standard" embedding should have the property that the points in each hyperplane generate that hyperplane, and form a minimal dependent set ("circuit"). Dually, the $k$ hyperplanes through each point may intersect precisely in that point, and so form a dual "circuit". But this condition is not always achievable.

Received 24th July, 2006

Copyright Clearance Centre, Inc. Serial-fee code: 0004-9727/07 \$A2.00+0.00. 
If it is embeddable the configuration can be called "geometrical", but note that this property usually depends upon the base field (or skew-field) that is chosen.

We have a slightly different notion of embedding to that in [2, Section 3.2]. There, they assume that the configuration is of rank 3; that is, possibly, embedded in a plane. Here, we try to embed the $n_{k}$ configuration as a rank $k$ matroid into $(k-1)$ dimensional space. We think that this is a more natural generalisation of $n_{3}$ configurations.

It is shown in Section 2 that the embedding problem reduces to that of finding a rooted spanning tree of the related Levi graph, and this can easily be found by using a well-known greedy algorithm. Although a "standard" embedding might not exist for a given field, if the base field is chosen large enough (for example, it is algebraically closed) and with the right characteristic, such embeddings should usually appear, although in some cases it can be shown that embeddings (with all the points and hyperplanes distinct) can never appear. (One of the $10_{3}$ configurations is a case in point.) A single algebraic equation can be found involving many free parameters. This generalises and explains a similar result for $n_{3}$ configurations for which various methods have already been found.

A "closed" $n_{k}$ configuration is one for which the last incidence (for example, using our spanning tree algorithm above) is always trivially satisfied. Naturally this might depend upon the field that is chosen. If this holds we call it a theorem, (or "Satz" in German). The complement of closed (standard) configurations ("theorems") is shown in Section 3 to be closed, so that we can show that the well-known Pappus and Desargues theorems in the plane, imply theorems in 5- and 6-dimensional space respectively as well. At present we know of $n_{k}$ theorems that have parameters $7_{3}, 7_{4}, 8_{4}, 9_{3}, 9_{6}$, $10_{3}$ and $10_{7}$. These are discussed in the later Sections, while the last Section contains some conjectures and ideas for further research in this subject. For each $n \geqslant 2$ there is also a 'degenerate' $n_{2}$ theorem, whose Levi graph (defined below) is a $2 n$-cycle. In the present discussion we assume that $k \geqslant 3$. The Pappus and Desargues configurations, in 2 or 3 dimensions, are only briefly dealt with, since they are so well known that a huge number of publications deal with their properties. However, geometrical diagrams and Levi graphs can be found in [5].

\section{Embedding Configurations into Projective Space}

The survey by Steinitz [12] contains reference to his algorithm that gives a method of embedding an $n_{3}$ configuration into a plane (over a field). A discussion and application of Steinitz's method appeared recently in [8]. Let us note that the points of the configuration are chosen in sequence so that the lines are then constructed from the points, and the lines have no independent freedom. 
Other methods have appeared (see [2, Section 3]; $[5,13]$ ) In particular, the author's method [5] was to use the Levi graph (defined below). It deletes a certain flag, that is, a point/line incidence $(P, l)$, from the graph, and then orders the points and lines so that the vertices (points and lines) lie at increasing distances from $P$ or $l$.

The common thread between all these algorithms is that all the points and lines can be put into the projective plane (with a certain freedom) so that $3 n-1$ incidences are satisfied. The last incidence is not automatic but in most cases the freedom of the configuration allows for the last incidence to be satisfied (since it is just the same as solving an algebraic equation in many variables).

When the configuration is a "theorem" then the last incidence should be automatically satisfied, while if the configuration is not embeddable, then the last incidence is satisfied only when the configuration degenerates by some of the points or lines becoming the same.

Here we can generalise and at the same time simplify things with a method that will produce all the above algorithms as special cases, while also doing it for the more general $n_{k}$ configurations in $(k-1)$-dimensional projective space.

First, we define the Levi graph of an $n_{k}$ configuration [10], (first lecture).

DEFINITION 2: A graph is an ordered pair $(V, E)$, where $V$ is a set of vertices and $E$ is a set of subsets of size 2 in $V$, called edges. A graph is bipartite if the vertices $V$ can be partitioned into 2 non-empty subsets such that only edges containing one vertex from each of the two subsets appear in $E$.

DEFINITION 3: The Levi graph of an $n_{k}$ configuration $C$ with point-set $P$ and block-set $B$ is the bipartite graph $(P \cup B, E)$, where $\{p, b\}$ is an edge if and only if $p \in P, b \in B$, and $p$ is incident with $b$; that is, $(p, b)$ is a flag of $C$.

Examples of Levi graphs of $n_{3}$ configurations appear in $[5,8]$.

NOTE 1. It is well-known that a given trivalent graph (having three edges through every vertex) is the Levi graph of an $n_{3}$ configuration if and only if it is bipartite and of girth at least 6 . (The girth is the size of the smallest circuit in the graph. For example, if the girth is 6 there is a triangle in the configuration.)

NOTE 2 . We consider only connected configurations now, since disconnected configurations can be split into independent smaller connected ones.

In [5] it was shown that the problem of embedding an $n_{3}$ configuration into the plane is equivalent to drawing the $2 n$ vertices and edges of the associated Levi graph (minus one edge) in the Euclidean plane so that there is one vertex at the bottom, (having 2 edges going up), and no other vertex has all 3 of its edges going up. Then the way to embed the configuration is to put the elements (points or lines) at the top of the graph into the plane (with the appropriate freedom which is 2 minus the 
number of edges going up from that vertex), and then to work one's way down the graph, eventually reaching the bottom. The final deleted edge of the graph then gives an algebraic condition for the embeddability of the configuration.

The method given in [5] to draw such a "well-ordered" Levi graph in the Euclidean plane was to start with the bottom vertex and then to put vertices of distance 1 just above it, then distance 2 above those, and so on.

Of course, any embedding algorithm, such as that of Steinitz, gives an alternative way of drawing a "well-ordered" Levi graph, although it may have additional favourable properties. For example, the well-ordered graph coming from Steinitz's algorithm has the additional property that for any line of the configuration, the corresponding vertex is always joined to two (point) vertices immediately above it. Thus the points of the configuration are the only elements able to have any positive freedom in the algorithm.

It is clear that similar processes can be used to embed the more general $n_{k}$ configurations into $(k-1)$-dimensional space. It is only necessary to draw a "well-ordered" Levi graph (with one edge missing) in the Euclidean plane, so that the only vertex with all edges going up is the one at the bottom (having $k-1$ edges, one being deleted). The freedom of a vertex is now given by the expression $k-1$ minus the number edges going up from that vertex. Then to actually embed the configuration we again start at the top of the "well-ordered" Levi graph and go down, leaving the final edge of the graph (or in other words the final flag of the configuration) as an algebraic equation to be solved.

We can now say precisely how to find "well-ordered" Levi graphs. First, we recall a definition from graph theory.

DEFINITION 4: A spanning tree of a (connected) graph with $v$ vertices is a set of $v-1$ edges containing no circuit: thus each vertex appears on at least one of the edges of the tree.

Note 3. A spanning tree is equivalent to a basis of the (binary and graphical) matroid which has, as set of points, the set of edges of the graph.

THEOREM 1. A way that always constructs any "well-ordering" of the Levi graph of an $n_{k}$ configuration is first to find a spanning tree $T$ of the graph. Then, select any vertex $R$ (or "root") of the tree. Next, label any vertex on the outside of the tree (a vertex having valency one) at the top. Delete that vertex from $T$, and repeat by finding another vertex on the outside of the tree. Put that vertex down from the first one, and repeat the loop, working inwards so that $R$ is the last remaining vertex. Finally, put $R$ at the bottom of the ordering.

PROOF: This algorithm produces a well-ordering because after each step the vertex that has been last put in order is connected in the tree $T$ to another vertex that has 
not been put into order. The only case where this does not happen is the final vertex $V$. The algorithm relies on the existence of a spanning tree, but this is easily found by a greedy algorithm as in matroid theory. (Just keep choosing edges so that no circuit is formed in the graph. Eventually a spanning tree arises.) Also, given any well-ordering of the graph, it is clear that it will come from some spanning tree, which can be constructed by choosing a sequence of vertices from the bottom of the ordering, subject to the condition that no circuit is formed.

\section{The Complementary $n_{k}$ Theorems}

DEFINITION 5: The complementary configuration $\bar{C}$ to an $n_{k}$ configuration $C:=(P, B, I)$ is the $n_{n-k}$ configuration $\left(P, B, I^{\prime}\right)$, where $p I^{\prime} b \Longleftrightarrow p / I b$. Equivalently, the incidence matrix of $\bar{C}$ is the complement of the incidence matrix of $C$. (An incidence matrix of an $n_{k}$ configuration $(P, B, I)$ is an $n \times n(0,1)$-matrix with rows corresponding to the points, and columns to the blocks, with a 1 in a position $(p, b)$ if and only if $p I b$, and 0 's otherwise.)

Recall from Section 1 that an $n_{k}$ "theorem" for a $(k-1)$-dimensional projective space over a given field (or skew-field), is an $n_{k}$ configuration such that the final incidence is automatically satisfied, when we embed the configuration in that space, for example, using the algorithm given in Theorem 1 of Section 2.

Examples of such "closed" configurations (or theorems) are the Fano $7_{3}$ in planes over fields of characteristic 2, Pappus $9_{3}$ for field planes, or Desargues for skew-field or field planes. Other examples are discussed in the remaining sections. However, now we can show how to obtain a "bonus" theorem from a given one.

First, for people not fully conversant with matroid theory, let us recall some basic notions, applied to the case of "representable" matroids, which are the linear structures induced by finite sets of points in a projective space over a field or skew-field $F$.

DEFinition 6: The matroid dual of a set $S$ of $m$ points (spanning $(k-1)$-dimensional projective space over $F)$ is a set $S^{\perp}$ of $m$ points spanning the $(m-k-1)$ dimensional projective space over $F$, unique up to linear collineations of that space. A way to obtain this set of points is to first write the homogeneous coordinates of the points of $S$ (with respect to any fixed basis) as the columns of a $k \times m$ matrix $M$ over F. Then the orthogonal space to the row space of $M$ (with the usual inner product) can be written as the row space of another matrix $N$, the columns of which give the points of $S^{\perp}$.

Note that there is a natural 1-1 correspondence between the $m$ points of $S$ and the $m$ points of $S^{\perp}$, given by the ordering of the columns of the two matrices $M$ and $N$. So it is possible to identify the points of the two sets in some sense. 
There are many well-known properties of the matroid dual. Perhaps the defining property is that a subset of $S$ is a basis, that is, maximal independent set, if and only if the complement of that subset corresponds to a basis of $S^{\perp}$. (This can be used to define the dual for matroids more general than representable ones.) However, we need some other related properties here. They can be proved quite easily from the idea of basis in the matroid.

We recall the concepts of "circuit" and "hyperplane". Assume as above that $S$ is a set of points of rank $k$ in $(k-1)$-dimensional projective space.

DEFINITION 7: A circuit of a matroid $S$ is a minimal dependent set of points; that is, a non-empty subset of $S$ for which the deletion of any point makes an independent set. (The fundamental theorem of geometry tells us that any circuit of size $s$ in a representable matroid is projectively equivalent to $\left\{e_{1}, \ldots, e_{s-1}\right\} \cup\left\{e_{1}+\cdots+e_{s-1}\right\}$, where $e_{i}$ is the $i$ 'th unit point, with all zero's, except a 1 in the $i^{\prime} t h$ position.) A hyperplane $h$ of $S$ is a subset of rank $k-1$, such that for any point $P$ of $S \backslash h$, the rank of $h \cup\{P\}$ is $k$, that is, $h$ is a maximal proper subspace of $S$. (Equivalently, any basis of the matroid induced on $S$ by the restriction to $h$ can be extended to a basis of $S$ by the addition of any point of $S \backslash h$.)

After these definitions we note the main matroid property needed for the following theorem. It is an easy exercise, found in many matroid theory books.

Note 4 . The complement of a circuit in a matroid $S$ corresponds to a hyperplane in $S^{\perp}$, and conversely, a hyperplane of $S$ corresponds to the complement of a circuit in $S^{\perp}$. Thus, a certain subset of $S$ is simultaneously a circuit and a hyperplane if and only if the complement of that subset is both a circuit and a hyperplane of $S^{\perp}$.

The matroid preliminaries above make the following result quite straight-forward.

TheOREM 2. Given an $n_{k}$ theorem $C$ about a $(k-1)$-dimensional projective space over $F$ (field or skew-field), the complementary $n_{n-k}$ configuration $\bar{C}$ is then a theorem for $(n-k-1)$-dimensional space over $F$.

Proof: Consider the configuration $C$, embedded in space, as a matroid. Then we can assume, by the freedom involved in the embedding, that each hyperplane of $C$ contains $k$ points which are a circuit (minimal dependent set). Thus, in the dual matroid $C^{\perp}$, the complementary set of $n-k$ points also is a hyperplane and simultaneously a circuit (from Note 4). But this means that $C^{\perp}$ and $\bar{C}$ are identical (combinatorial) $n_{n-k}$ configurations. Now if $\bar{C}$ were not a theorem then it would be possible to construct it in the $(n-k-1)$-dimensional space so that all the circuit/hyperplanes existed except one. Then we would get a contradiction to the theorem $C$ when we took the dual. So $\bar{C}$ must be a theorem.

NOTE 5. The geometrical dual (by interchanging points and hyperplanes, or equiva- 
lently by taking the transpose of an incidence matrix) of an $n_{k}$ theorem is also an $n_{k}$ theorem.

DEFINITION 8: The "freedom" of an $n_{k}$ configuration $C$ is the dimension of the algebraic variety of all embedded configurations of $C$ in the corresponding space, modulo the group of collineations of that space.

THEOREM 3. The freedom of a general embeddable $n_{k}$ configuration is

$$
f(n, k):=2 n(k-1)-n k-\left(k^{2}-1\right)=(n-k) k-2 n+1=n(k-2)-k^{2}+1 .
$$

If the $n_{k}$ configuration is a theorem then the freedom is $n(k-2)-k^{2}+2$.

Proof: The general freedom of $2 n$ points/hyperplanes is $2 n(k-1)$ since they are in $(k-1)$-dimensional space. This is reduced, firstly by the $n k$ incidences that must be satisfied, and secondly by the dimension of the group of (linear) collineations of that space, which is $k^{2}-1$. For an $n_{k}$ "theorem" there is one incidence that is not counted, and this adds 1 to the freedom.

For example, an $n_{3}$ configuration in the plane has freedom $n-8$. If it is a theorem then the freedom is $n-7$.

Note 6. An $n_{k}$ and its complementary $n_{n-k}$ should have the same freedom, since there is a 1-1 correspondence between their embeddings in their respective spaces, modulo the collineation groups of those spaces. This is proved once we check that

$$
f(n, k)=n(k-2)-k^{2}+1=f(n, n-k)=n(n-k-2)-(n-k)^{2}+1 .
$$

Note 7. The geometrical dual, and complement of an $n_{k}$ configuration, all have isomorphic automorphism groups.

The next sections are brief summaries of the known $n_{k}$ theorems. For each configuration $C$ we denote the size of the group of automorphisms by $\mid$ Aut $(C) \mid$.

\section{FANO 73 AND ITS COMPLEMENT}

The "Fano" $7_{3}$, or in other words, the projective plane of order 2 , is a theorem for planes over fields of characteristic 2 . The complementary $7_{4}$ configuration is a theorem for 3-dimensional space of characteristic 2 . It is naturally embedded in $P G(3,2)$ by the deletion of a point $P$ and 7 points of a plane $\pi$ not through $P$. Then the 7 planes of the $7_{4}$ are those that are not passing through $P$ and not equal to $\pi$. The freedom of Fano (and its complement) is -1 , indicating that it cannot be normally embeddable in a plane, without it being a theorem.

By Theorem 3, for fields of characteristic 2, $f($ Fano $)=0$, or -1 otherwise. $\mid$ Aut $($ Fano $) \mid=168$. 


\section{PAPPUS $9_{3}$ AND its Complement}

The $9_{3}$ theorem is due to Pappus of Alexandria, circa $340 \mathrm{CE}$. It is a theorem for planes over fields. By Theorem 2 the complementary $9_{6}$ is also a theorem for 5-dimensional space over a field.

Theorem 4. The Pappus 96 in 5-dimensional space over a field lies on the Veronese surface, and any embedding can be obtained by using the natural correspondence between the points of an embedding of the $9_{3}$ in the plane, and mapping via the Veronese mapping $(x, y, z) \mapsto\left(x^{2}, y^{2}, z^{2}, y z, x z, x y\right)$ onto the Veronese surface.

Proof: The above mapping onto the Veronese surface (see [9] for many properties of these surfaces and generalisations to higher spaces) has the property that the complement of any line, that is, 6 points on two other disjoint lines of the $9_{3}$, lie on a hyperplane of 5-dimensional space. This is because all the points on a line of the plane are mapped to a "conic-plane" of the surface, and any pair of conic-planes intersect in the point corresponding to the point of intersection of the pair of lines in the original projective plane. Thus the Veronese mapping does indeed take any Pappus $9_{3}$ of the plane to a Pappus 96 of the 5-d space. By looking at the freedom of the configuration now, we see that the freedom of the $9_{6}$ 's lying on the Veronese surface is the same as the freedom of the $9_{3}$ 's in the plane. Thus as algebraic varieties the freedom of general Pappus 96 's in 5-d space is the same as those on a Veronese surface. This shows that the two algebraic varieties of 96 's are the same, and every Pappus $9_{6}$ lies on a Veronese surface. (Another proof can be obtained by knowing that any sufficiently general set of 9 points in 5 -d space lies on a Veronese surface $V^{2}(2)$; see the following Lemma.)

Let $V^{i}(k-1)$ denote the Veronesean or Veronese variety of hypersurfaces of degree $i$ in $(k-1)$-dimensional projective space over a field. $V^{i}(k-1)$ is a variety of dimension $k-1$ in $\left(\left(\begin{array}{c}i+k-1 \\ i\end{array}\right)-1\right)$-dimensional projective space.

LEMMA 1. ([6]) The number of points that determine the Veronesean $V^{i}(k-1)$ is $\left(\begin{array}{c}i+k-1 \\ i\end{array}\right)+k$.

Proof: The number of parameters of the group of the space in which the Veronesean is embedded is $\left(\begin{array}{c}i+k-1 \\ i\end{array}\right)^{2}-1$, while the number of parameters of the group of the Veronesean itself is the same as that of the projective geometry of dimension $k-1$, which is $k^{2}-1$. To say that a variety of dimension $k-1$ lies on a specified point of $n$-dimensional space is to restrict that variety by $n-k+1$ parameters. Hence, the number of points needed to uniquely determine the Veronese variety of index $i$ 
corresponding to $S_{k-1}$ is

$$
\frac{\left(\begin{array}{c}
i+k-1 \\
i
\end{array}\right)^{2}-k^{2}}{\left(\begin{array}{c}
i+k-1 \\
i
\end{array}\right)-k}=\left(\begin{array}{c}
i+k-1 \\
i
\end{array}\right)+k .
$$

Here we give a direct algebraic proof of the Pappus $9_{6}$ theorem. Firstly, label the 9 points of the configuration $1,2,3,4,5,6, a, b, c$, where $12345 a, 13456 b, 12456 c, 2346 a b$, $2356 a c, 1236 b c, 456 a b c, 124 a b c$, and $135 a b c$ are the 9 block-hyperplanes. We have omitted some commas in the following to make things more concise. Note that $a b c$ is chosen so that it corresponds to a triangle contained in the planar Pappus $9_{3}$ (that is, a basis). Thus the complement 123456 is a basis of the $9_{6}$ (see Section 3), which can be coordinatised in the natural way by identifying 1 with the unit vector $e_{1}, 2$ with $e_{2}$, et cetera.

In terms of the three points $a b c$ the conditions that the points of each blockhyperplane are dependent (in general forming a circuit) are that:

(1) $a \in\langle 12345\rangle$,

(2) $b \in\langle 13456\rangle$,

(3) $c \in\langle 12456\rangle$,

(4) $\langle a b\rangle \cap\langle 2346\rangle \neq \emptyset$,

(5) $\langle a c\rangle \cap\langle 2356\rangle \neq \emptyset$,

(6) $\langle b c\rangle \cap\langle 1236\rangle \neq \emptyset$,

(7) $\langle a b c\rangle \cap\langle 456\rangle \neq \emptyset$,

(8) $\langle a b c\rangle \cap\langle 124\rangle \neq \emptyset$,

(9) $\langle a b c\rangle \cap\langle 135\rangle \neq \emptyset$.

From $(1-3)$ we can write

$$
\begin{aligned}
& a=\alpha 1+d 2+e 3+\gamma 4+\beta 5 \\
& b=\alpha 1+g 3+\delta 4+\beta 5+h 6 \\
& c=\alpha 1+i 2+\gamma 4+\phi 5+j 6
\end{aligned}
$$

In this notation we are using the digits $1, \ldots, 6$ as coordinates, and the $\alpha, d, e$, $\gamma, \beta, g, \delta, h, i, \phi, j$ are variables. The latter are also non-zero because the 6 points in each of the first three block-hyperplanes are circuits. The equality of the various coefficients, $\alpha, \gamma$ and $\beta$ in the three equations above can be obtained from items (4) and (5) above, because we can assume that $a-b$ is in $\langle 2346\rangle$ (that is, the coefficients of coordinates 1 and 5 must be dependent) and $a-c$ is in $\langle 2356\rangle$ (that is, the coefficients of 1 and 4 must also be dependent).

Further, (6) gives the condition that $\delta \phi=\gamma \beta$, (because the coefficients of 4 and 5 must be dependent). 
For the remaining conditions we have the following implications:

$$
\begin{aligned}
& (7) \Longrightarrow-g i+i e+d g=0 \\
& (8) \Longrightarrow e \beta j-e h \phi-g \beta j=0 \\
& (9) \Longrightarrow d \delta j-d h \gamma+i \gamma h=0
\end{aligned}
$$

These hold because the $3 \times 3$ determinants for the three equations $(7-9)$, corresponding to the restriction to columns 123,356 , and 246 , must be zero.

In order to verify that the Pappus complement is a theorem we must show that the four equations resulting from $(6-9)$ above are dependent.

Firstly, (7) implies that $d g=i(g-e)$, while (9) implies that $d(\delta j-h \gamma)=-i \gamma h$. Multiplying this new (9) by $g$ we obtain $g d(\delta j-h \gamma)=-g i \gamma h$. Replacing $g d$ by $i(g-e)$ means that we can cancel $i$ 's on both sides, giving us $(g-e)(\delta j-h \gamma)=-\gamma h g$. Simplifying, we obtain $(g-e) \delta j+e h \gamma=0$ which implies that $g \delta j=e(\delta j-h \gamma)$.

Now (8) is the same as $e(\beta j-h \phi)=g \beta j$. Multiplying both sides by $\delta$ implies that $\delta e(\beta j-h \phi)=\delta g \beta j$, and substituting $g \delta j$ by $e(\delta j-h \gamma)$ we obtain $\delta e(\beta j-h \phi)$ $=\beta e(\delta j-h \gamma)$. Cancelling $e$ 's on both sides, then simplifying there holds $-\delta h \phi$ $=-\beta h \gamma$, and after dividing out by $h$ we obtain the residual equation $\delta \phi=\gamma \beta$, which is equation (6). This tells us that equation (6) is dependent upon the others, and that the Pappus $9_{6}$ is a theorem.

For all fields, $f($ Pappus $)=9-7=2$, and for skew-fields it is 1. $\mid$ Aut (Pappus) $=108$. Pappus is also geometrically self-dual.

Note. We say that a projective plane is Pappian if Pappus theorem is satisfied in it. We know that Pappian planes are those coordinatised by fields.

\section{Desargues $10_{3}$ and its Complement}

Desargues $10_{3}$ configuration $\mathbb{D}$ was discovered in the 17 th century CE. It is a theorem easily proved from the incidence properties of 3-dimensional projective space (see for example [1]), and so it is valid for all planes over skew-fields. The complementary $10_{7}, \mathbb{D}^{c}$, will also be a theorem for any 6 -dimensional projective space.

$\mathbb{D}$ can be represented using subsets of size 2 in a set of size $5, S:=\{1,2,3,4,5\}$, as follows. The points of $\mathbb{D}$ are the 10 members of the set $P:=\{\{i, j\} \mid i, j \in S, i \neq j\}$. Let us write each subset $\{i, j\}$ more concisely as $i j(=j i)$. The blocks of $\mathbb{D}$ are the 10 subsets $l_{i j k}:=\{i j, j k, i k\}$, where $i \neq j \neq k \neq i$. Then $\mathbb{D}^{c}$ is the structure with the same set $P$ of points, but with blocks which are the complements of the blocks of $\mathbb{D}$ : that is, $b_{i j k}:=P \backslash l_{i j k}$.

Suppose that the 10 points and 10 blocks $b_{i j k}$, now hyperplanes, of $\mathbb{D}^{c}$, are embedded in 6-dimensional space. Each block $b_{i j k}$ is a circuit, and so any proper 
subset of it is an independent set of points. In particular there are 5 subsets of $P$ of 4 points each: viz. $T_{i}:=\{i j \mid j \neq i\}$, which are independent, and so they each generate a 3-dimensional subspace $\left\langle T_{i}\right\rangle$. For $i \neq j$ the union $T_{i} \cup T_{j}$ is the block $b_{p q r}$, where $\{p, q, r\}=S \backslash\{i, j\}$. Note that by Grassmann's formula for dimensions of joins and intersections of two subspaces, since $3+3=1+5, T_{i} \cap T_{j}=\{i j\}$ but $\left\langle T_{i}\right\rangle \cap\left\langle T_{j}\right\rangle$ is a line $t_{i j}$, while $\left\langle T_{i}\right\rangle \cup\left\langle T_{j}\right\rangle=\left\langle b_{p q r}\right\rangle$ is a 5 -dimensional subspace. We can show below that these 10 "tangent lines", $t_{i j}$, all pass through a common point.

Consider, for any $1 \leqslant i \leqslant 5$, the intersection of the 4 block-hyperplanes $b_{p q r}$, where none of $p, q, r$ is equal to $i$. Then the intersection contains the 4 points of $T_{i}$ and so a 3-d subspace. This shows that these 4 hyperplanes are dependent, because 4 independent hyperplanes of 6-dimensional space always intersect in a plane. Dualising (with the geometrical dual), the 10 block-hyperplanes become a set of 10 points of 6-dimensional space, such that there are 5 subsets of 4 points each, which form circuits in 5 planes (since the geometrical dual of a 3 -dimensional space is a plane). The next lemma analyses the properties of such a set of 5 planes.

Lemma 2. A set of 5 planes, pairwise intersecting in 10 points, with precisely 2 planes through any point, and with no three of the 10 points collinear, generates at most a 5-dimensional subspace.

Proof: We may assume that 4 of the planes intersect pairwise in 6 points that are independent. Then the remaining plane intersects each of the first 4 planes in points that generate that plane. Hence the fifth plane is contained in the 5-dimensional space generated by the other 4 planes.

We assume that any three block-hyperplanes intersect in at most a 3-dimensional subspace, that is, that when dualising the three points are not collinear. (This appears to be a reasonable condition for a general embedding of $\mathbb{D}^{c}$ and it can be justified directly by matroid duality.) The five 3 -spaces $\left\langle T_{i}\right\rangle$ dualise geometrically to five planes satisfying the conditions of Lemma 2 above, and this shows that the 10 points, corresponding to the geometrical duals of the block-hyperplanes, actually generate a 5 dimensional subspace: that is, the block-hyperplanes all pass through a common point $N$, which we call the nucleus of $\mathbb{D}^{c}$.

Thus we have:

THEOREM 5. The 10 block-hyperplanes of an embedding the complementary Desargues theorem $\mathbb{D}^{c}$ in 6-dimensional space (assuming the most general position of the points and hyperplanes), all pass through a common point $N$ that is not equal to any of the 10 points of $\mathbb{D}^{c}$.

COROLLARY 1. The general embedding of $\mathbb{D}^{c}$ in 6-dimensional space can never be self-dual. More specifically, the geometrical dual of the configuration is actually in 
a 5 -dimensional subspace.

We can confirm these properties most easily by considering the Desargues configuration embedded in 3-dimensional space. As in the 2-dimensional embedding, there will be 10 points and 10 lines, labelled similarly, but there are also 5 planes, each containing 6 points on 4 of the lines: that is, the points that are not in $T_{i}$, for $1 \leqslant i \leqslant 5$. Since planes are the same as hyperplanes in 3-dimensional space the subsets of 4 points, $T_{i}$, will be circuits in the 5 -dimensional matroid dual of the 3 -dimensional embedding. Thus these $T_{i}$ will be 5 planes that pairwise intersect in a point in 5 dimensions.

Any planar Desargues configuration is the projection of a 3-dimensional configuration from some point $M$ of that 3-space. Hence we consider the matroid dual of the 11 points formed by $M$ and that Desargues in 3-dimensional space. We obtain 11 points of an 11-4-1=6-dimensional space such that the deletion of the corresponding point $N$ (corresponding to $M$ ) is the configuration $\mathbb{D}^{c}$. Note that deletion and projection are dual concepts in matroid theory. The block-hyperplanes of $\mathbb{D}^{c}$ (passing through $N$ ) are complementary to the lines of Desargues in the 3-dimensional embedding, which are circuits. This confirms the fact the block-hyperplanes are all passing through $N$. Also, the 5 planes of the 3 -dimensional embedding containing 6 points each, are complementary to the sets $T_{i} \cup\{N\}$, which must be all circuits of 5 points in 6-dimensional space. This also confirms that the corresponding 3-spaces $\left\langle T_{i}\right\rangle$ all pass through $N$, which forms the fifth point with $T_{i}$ for the circuit. Thus we indeed have tangent lines passing through each point of $\mathbb{D}^{c}$, and also the nucleus point $N$.

There are many other properties that can be found but let us finish this discussion of $\mathbb{D}^{c}$ by noting several ways to get a set of 5 planes in 5 -dimensional space, pairwise intersecting in a point. Both of these ways involve the Veronese surface $V:=V^{2}(2)$; see [9] for a discussion of general properties of $V$. (It is necessary to assume that the characteristic of the field is not two, because the Veronesean looks different in this case.)

First, we consider 5 points of the plane, no 3 collinear (forming a " 5 -arc"). The repeated lines through each of the 5 points form 5 conics in planes on $V$ (called conic planes). These planes will pair-wise intersect in a point.

Secondly, we consider 5 lines of the plane, no 3 concurrent (forming a "dual 5arc"). Each of these lines, multiplied by a general line of the plane, are mapped by the Veronese mapping to a point of the cubic hypersurface $\Omega$ attached to $V$ (corresponding to the degenerate conics in the plane). These points form 5 planes, also pairwise intersecting in a point. As in Lemma 2 above, the 5 planes lie in a 5 -dimensional space, while the set of 10 points formed by the pair-wise intersections of the planes is the matroid dual of a Desargues configuration in 3-space.

An interesting problem is to determine when the 10 points of the matroid dual (in 5 dimensions) of Desargues in 3 dimensions lies on a Veronese surface. Another 
problem is to determine when a dual $k$-arc of lines of the plane, mapping to a set of $k$ tangent planes of a Veronese surface, are also $k$ conic planes of another Veronese surface. (We conjecture that it happens when the $k$ lines of the dual $k$-arc lie on a dual conic. Notice that 5 general points in the plane determine a unique conic.)

The freedom of Desargues embedded in 3 dimensions can be calculated as follows. A general tetrahedron of 3 -space has freedom $4.3=12$. By taking a general plane of the space (of freedom 3), one produces Desargues by adjoining the 4 points of the tetrahedron to the intersections of its 6 edges with the plane. Since the group of 3space has freedom 15 this shows that the freedom of Desargues (reduced as usual by the group) is $12+3-15=0$. Thus there is basically a unique (or at least finite) number of classes of 3-dimensional Desargues configurations in space up to the group of collineations. Since the 5 planes, pairwise intersecting in a point, are the matroid dual of Desargues in 3 dimensions, they also have freedom 0 , and must be conic-planes (or tangent planes) of some Veronese surface.

Thus, we expect that every embedding of the Desargues $10_{7}$ in 6-dimensional space is on a cone, with vertex that is the nucleus of that configuration, and base which is a Veronese surface.

$f($ Desargues $)=10-7=3$, while $\mid$ Aut (Desargues $) \mid=120$.

Indeed, Aut (Desargues) $\cong S_{5}$. Desargues $10_{3}$ is geometrically self-dual, although surprisingly the $10_{7}$ is only combinatorially self-dual, and can never be geometrically self-dual; see Corollary 1.

Note. We say that a projective plane is Desarguesian if Desargues theorem is satisfied in it. We know that Desarguesian planes are those coordinatised by skew-fields.

\section{MÖBIUS $8_{4}$}

In 1828 Möbius [11] discovered his $8_{4}$ theorem. It is self-complementary, which can be seen from an incidence matrix such as

$$
\left(\begin{array}{cc}
I & J-I \\
J-I & I
\end{array}\right)
$$

where $I$ and $J$ are the $4 \times 4$ identity and all 1 's matrices respectively. Often it is described as two tetrahedrons being circumscribed simultaneously about the other.

Using the above incidence matrix let us label the 8 points of the $8_{4}$ from 1 up to 8. Then the blocks are: $1678,2578,3568,4567,2345,1346,1247,1238$.

One of the best ways to construct the configuration in 3-d space is to start with a quadrangle of 4 points (labelled $1,6,7,8$ ) in a plane $\alpha$, with a line $l$ of $\alpha$ not passing through the 4 points. The 6 secants of the quadrangle (joining the pairs of points) intersect $l$ in 6 points of an "involution"; see [1, Volume I, Section 1, Exercise 10, 
p. 61]. Thus let 17 intersect $l$ in $a, 68$ in $b, 18$ in $c, 67$ in $d, 16$ in $e$, and 78 in the point $f$ on $l$. (The involution induced on $l$ is actually the one taking $a \leftrightarrow b, c \leftrightarrow d$ and $e \leftrightarrow f$.)

Now construct another plane $\beta$ through $l$ containing a similar quadrangle of 4 points (labelled $2,3,4,5$ ) such that the 6 secants of this quadrangle determine the same 3 pairs of two points in involution on $l$. We do this by letting 24 intersect $l$ in $a, 35$ in $b, 23$ in $c, 45$ in $d, 34$ in $e$, and 25 in $f$. (Notice that this is a kind of "complementary" association of the pairs of the quadrangle $2,3,4,5$ with the involution in comparison to the association with $1,6,7,8$. It is not achieved by a 1-1 correspondence, or projection, between the points of the two quadrangles.)

This can be done if the plane is Pappian. The union of the two quadrangles is then the set of points of the $8_{4}$, while the two planes $\alpha$ and $\beta$, and the 6 planes that are generated by the corresponding pairs of secants of the two quadrangles, that intersect on $l$, comprise the 8 planes of the $8_{4}$.

Thus the Möbius $8_{4}$ is a theorem for 3-dimensional projective space over a field. An alternative proof (not using Pappus or the idea of involutions coming from quadrangles) is to note that this $8_{4}$ has 4 pairs of disjoint planes which contain 4 points. Thus there are 4 degenerate plane-pair quadrics through the 8 points. Now the dimension of the space of quadrics in 3-d space is 9 . Thus 9 general points determine a given quadric and so 8 general points determine a pencil of quadrics. But we have actually a net (2-dimensional space) of quadrics through the 8 points. By Bezout's theorem three general quadrics intersect in $2^{3}=8$ points. Following from this we may use $[1$, Volume IV, Section 1, Exercise 12, p. 61], where it is stated:

"In space of $n$ dimensions, all $(n-1)$-dimensional quadrics which pass through $(1 / 2) n(n+1)+1$ general points are expressible linearly, when their equations are in point-coordinates, by $n$ linearly independent quadrics passing through these points; all such quadrics, therefore, pass through $2^{n}-1-(1 / 2) n(n+1)$ other points."

Thus, in the case $n=3$, we have that quadrics passing through 7 points, also pass through an eighth point. Using this, it is clear that the last incidence of the Möbius $\mathbf{8 4}$ is equivalent to the fact that the degenerate quadric consisting of one of the 4 planepairs, passes through all 8 points, which indeed follows by the algebraic method as stated in [1].

As noted in [1, Volume IV, Section 1, pp. 18-20], Wallace's theorem of 1806 ([14]) is equivalent in some sense to Möbius $8_{4}$. We recall Wallace's theorem: given any 4 lines of a quadrilateral in a plane over a field, any 3 of these lines determine a triangle of points and a circle that passes through these points. (A "circle" in a general Pappian projective plane is really a non-degenerate conic that passes through two fixed points on a line not passing through any of the 6 points of intersection of the lines of the 
quadrilateral.) Now we have 4 "circles" passing through the 4 triangles associated with the quadrilateral. Then Wallace's theorem says that these 4 circles pass through a common point.

A way to associate the $8_{4}$ with Wallace's theorem is as follows. It is well known that the set of circles and lines of an affine plane (projective plane minus a fixed line) is the same structure as the Möbius plane, which is the set of non-tangent plane intersections with an elliptic quadric (or "sphere") in 3-dimensional projective space. The lines of the affine plane correspond to those plane sections passing through a fixed point on the sphere, and the circles are all the other non-tangent plane sections. Thus, transforming Wallace's configuration of 4 lines and 4 circles through 7 points in the plane we see that there is a further point on the sphere through which 4 circles pass. It is easily checked that the Wallace and Möbius configurations are the same with this transformation. The fact that the Möbius $8_{3}$ lies on an elliptic quadric is obvious, once we know that there is a large enough set of quadrics passing through the set of 8 points. (See the alternative proof of the theorem via quadrics in the paragraph above.)

Note 8. Miquel's theorem (see [1, Volume IV, Section 1], or [3, Section 6]), is a theorem about 6 circles and 8 points on an elliptic quadric (or "sphere"). The configuration is isomorphic to the 8 vertices and 6 sides of a cube. Clearly it is the same as the Möbius $8_{4}$ with one set of 2 disjoint planes omitted.

For all fields, $f$ (Möbius) $=2$, and for skew-fields it is 1 . $\mid$ Aut (Möbius) $\mid=8.24$ $=192$. It is geometrically self-dual and self-complementary.

\section{Configurations That Are Not Embeddable}

There are several different types of $n_{k}$ configurations in this list. We omit discussion of configurations that are not embeddable due to compulsory extra incidences. For example, Grünbaum's $16_{3}$, see [7, Figure 8]. Some configurations are not embeddable because the space is just too small (not enough points in a finite space, for example), or the "final" algebraic equation expressing the incidence of the last flag is not solvable over the particular field. For example, the $8_{3}$ is not embeddable in the real plane, but it is in the complex plane.

Other than these types of situations there are certain configurations that are never embeddable, no matter which field extension is taken, and we list the known ones here.

The known $n_{3}$ configurations of this type are:

(1) the Fano $7_{3}$, in planes of characteristic not 2;

(2) one of the 10 different $10_{3}$ configurations for planes over a field; see [4].

Taking the complementary configurations we obtain:

the non-embeddable $7_{4}$, for 3 -spaces over a field of characteristic not 2 ;

and a non-embeddable $10_{7}$ for any 6-dimensional space over a field. 


\section{FURTher COMMENTS}

Here we note a few general directions in which to make progress.

A. Find more $n_{k}$ theorems. A good place to look first would be in 4-dimensional space, since no $n_{k}$ theorems are known there. For example, we conjecture that there are no more $n_{3}$ theorems to be discovered. Certainly we can show this when the $n_{3}$ contains a triangle.

B. Find more $n_{k}$ configurations that are not embeddable. From the preceding Section we see that the list of these is very short at present.

C. Classify $n_{k}$ configurations/theorems that are self-complementary and geometrically self-dual.

D. Find more relationships between $n_{k}$ configurations and algebraic geometry, such as the fact that the Pappus $9_{6}$ lies on a Veronese surface.

E. Find connections between $n_{k}$ theorems in different dimensions. For example, Pappus and Möbius could be related in a more direct manner.

F. Find more $n_{k}$ "theorems" of incidence other than those of Desargues and its complement (that are valid for skew-fields). In this regard it would be interesting to find a direct proof using the properties of higher dimensional spaces of the Desargues $10_{7}$ in 6 dimensions.

G. Given two Veronese surfaces $V_{1}$ and $V_{2}$ of 5 -dimensional space, investigate the intersections of the set conic planes of $V_{1}$ with the tangent planes of $V_{2}$.

We conjecture that that maximum intersection is reached when the conic planes correspond to the points of a non-degenerate conic of the plane, or the tangent planes correspond to the lines of a non-degenerate dual conic of the plane. Related to this is the fact that any 5 planes, each pairwise intersecting in a point (otherwise in general position in 5-space), are in the set of conic-planes of some Veronese surface, and at the same time in the set of tangent planes of another Veronese surface.

H. Investigate generalisations of Desargues theorem et cetera.

For $k \geqslant 2$ consider a collection of $k+2(k-1)$-dimensional subspaces of $\left(\left(\begin{array}{c}k+1 \\ 2\end{array}\right)-1\right)$-dimensional space such that each pair of subspaces intersect in a point. Thus there are $\left(\begin{array}{c}k+2 \\ 2\end{array}\right)$ points of intersection. Then it follows quite quickly that the matroid dual is a set of $\left(\begin{array}{c}k+2 \\ 2\end{array}\right)$ points of space of $k$ dimensions having a set of $\left(\begin{array}{c}k+2 \\ 3\end{array}\right)$ lines of 3 points each. For example, when $k=2$ the latter configuration is the Pasch axiom for projective space consisting of 4 coplanar lines and their 6 points of intersection (a matroid self-dual configuration). When $k=3$ it is Desargues configuration in $3 d$-space. The linear space of points and lines above in $k$-dimensional space may 
be represented combinatorially by the set of unordered pairs $a b$ of size 2 as "points", with $a$ and $b$ in a set of size $k+2$, with lines $\{a b, a c, b c\}$. Thus for $k>3$ it contains $\left(\begin{array}{c}k+2 \\ 5\end{array}\right)$ Desargues sub-configurations.

\section{REFERENCES}

[1] H.F. Baker, Principles of geometry I-IV (Cambridge University Press, Cambridge, 1922-1925).

[2] J. Bokowski and B. Sturmfels, Computational synthetic geometry, Lecture Notes in Math. 1355 (Springer-Verlag, Heidelberg, 1989).

[3] P. Dembowski, Finite geometries (Springer-Verlag, New York, 1968).

[4] D.G. Glynn, 'On the anti-Pappian $10_{3}$ configuration and its construction', Geom. Dedicata 77 (1999), 71-75.

[5] D.G. Glynn, 'On the representation of configurations in projective spaces', J. Statist. Plann. Inference 86 (2000), 443 456.

[6] D.G. Glynn, 'Transformations of codes and geometry related to Veroneseans', (preprint, see http://internal.maths.adelaide.edu.au/people/rcasse/DavidGlynn/papers.html).

[7] H. Gropp, 'Configurations and their realization', Discrete Math. 174 (1997), 137-151.

[8] B. Grünbaum, '3-connected configurations $\left(n_{3}\right)$ with no Hamiltonian circuit', Bull. Inst. Combin. Appl. 46 (2006), 15-26.

[9] J.W.P. Hirschfeld and J.A. Thas, General Galois geometries (Clarendon Press, Oxford, 1991).

[10] F.W. Levi, Finite geometrical systems, (six public lectures by the Hardinge Professor) (University of Calcutta, Calcutta, 1942).

[11] A.F. Möbius, J. f. Math. 3 (1828), 276. Werke 1 (1885), 437-443.

[12] E. Steinitz, 'Konfigurationen der projektiven Geometrie', Encyklopädie der Mathematischen Wissenschaften, mit Einschluss ihrer Anwendungen, Vol. 3, Part 1, 1st Half: Geometrie (1910), 481-516.

[13] B. Sturmfels, 'Computational algebraic geometry of projective configurations', J. Symbolic Comput. 11 (1991), 595-618.

[14] W. Wallace (1806), See Camb. Phil. Proc., Vol. XXI, 1923, 348.

School of Mathematical Sciences

University of Adelaide

Adelaide SA 5005

Australia

e-mail: david.glynn@adelaide.edu.au

dglynn@mac.com 